\title{
Frequent representations
}

\author{
V.I. ARNOLD *
}

\section{Introduction}

Given a unitary representation $T$ of a finite group $G$ in $\mathbb{C}^{n}$, write $M$ for the variety of such representations which are unitary equivalent to $T$.

Definition 1. The representation $T$ is said to be frequent if the dimension of the variety $M$ is maximal (among all representations of $G$ in the same complex space).

The study of the multiplicities of irreducible representations, forming the frequent one, had been started in [1]. We prove below that the irreducible representations are distributed, in the frequent representation (of large dimension), asymptotically in the same way as in the fundamental representation in the space of functions on $G$ : the frequencies of the irreducible components are proportional to their dimensions.

This result is always true asymptotically and is literally true if the dimension $n$ is divisible by the number of elements of the group. It might also be considered as the weak asymptotics

in the sense of [2], that is, as the average distribution for the variable values of the large dimension $n$.

I had observed the distribution of frequencies of representations described above, studying the symmetries of the eigenfunctions of problems in magneto hydrodynamics (see [3] and [4]). There, I have described the distribution of the higher eigenvalues whose eigenfunctions have different symmetries, the symmetry frequency being asymptotically proportional to the dimension of the representation space.

This phenomenon might be explained by the description of the oscillations, using the symmetry group of the fundamental domain. The oscillations in the fundamental domain can be continued to the whole oscillating manifold by the symmetry group. As a result of this, an eigenfunction, accordingly transformed to an irreducible representation, is occurring together with its continued versions. Therefore it is occurring together with several orthogonal copies of the isomorphic irreducible representation spaces of the eigenfunctions having the same symmetry, the number of these copies being equal to the dimension of the representation space (since it is the case for the fundamental representation).

I would like to thank R. Uribe-Vargas who was kind enough to type this text for me.

*Partially supported by RFBR, grant 02-01-00655. 


\section{Dimension of the variety of equivalent representa- tions}

Suppose that a finite dimensional unitary representation $T$ is the orthogonal sum of $s$ irreducible representations $T_{k}$ in spaces $\mathbb{C}^{a_{k}}$, each representation $T_{k}$ being repeated $p_{k}$ times, the complex dimension of the representation $T$ being

$$
n=\sum p_{k} a_{k}
$$

We shall study the choice of the multiplicities $p_{k}$, making maximal the dimension of the variety of representations which are unitary equivalent to $T$ (in the same space $\mathbb{C}^{n}$ ).

Theorem 1. The real dimension $D$ of the variety of representations which are unitary equivalent to $T$ in $\mathbb{C}^{n}$, equals

$$
D=n^{2}-\sum p_{k}^{2}
$$

Proof. Represent $\mathbb{C}^{n}$ as the orthogonal sum of the spaces $\mathbb{C}^{m_{k}}, m_{k}=p_{k} a_{k}$, where the different irreducible representations $T_{k}$ are realized.

Lemma 1. The complex dimension, $d$, of the manifold of representations of the Hermitian space $\mathbb{C}^{n}$ in the form of an orthogonal direct sum of s subspaces of complex dimensions $m_{k}$ is equal to the value of the second basic symmetric function,

$$
\sigma_{2}\left(m_{1}, \ldots, m_{s}\right)=m_{1} m_{2}+\cdots+m_{s-1} m_{s}
$$

Proof. The decompositions $\mathbb{C}^{a}+\mathbb{C}^{b}$ form the Grassman manifold of complex dimension $a b=\sigma_{2}(a, b)$.

The complex dimension $d_{s}$ of the manifold of decompositions in the form of $s$ summands can be calculated inductively according to the following fact:

$$
d_{s}=m_{s}\left(n-m_{s}\right)+d_{s-1}=m_{s}\left(m_{1}+\cdots+m_{s-1}\right)+d_{s-1}=\sigma_{2}\left(m_{1}, \ldots, m_{s}\right),
$$

provided that $d_{s-1}=\sigma_{2}\left(m_{1}, \ldots, m_{s-1}\right)$.

To obtain (from proved Lemma 1) the real dimension of the variety of unitary representations equivalent to $T$, one should add to $2 d_{s}$ the sum of the dimensions of the manifolds of reducible representations in $\mathbb{C}^{m_{k}}$, which are $p_{k}$-fold multiples of the irreducible representations $T_{k}$.

Lemma 2. The real dimension of the manifold of unitary representations in $\mathbb{C}^{m}, m=p a$, which are unitary equivalent to the p-fold multiple of the irreducible representation $R$ in $\mathbb{C}^{a}$ is equal to

$$
r=(p a)^{2}-p^{2}
$$


Proof. The unitary group of $\mathbb{C}^{n}$ has real dimension $m^{2}=p^{2} a^{2}$. To get $r$, we should substract the dimension of the stationary subgroup of the given $p$-fold version $R^{\prime}$ of the irreducible representation $R$,

$$
\left\{A \in U(m): A R^{\prime}=R^{\prime} A\right\} .
$$

The description of this subgroup $\{A\}$ is provided by Shur Lemma. Denote by $i_{k}: \mathbb{C}^{a} \rightarrow$ $\mathbb{C}^{m}$ the natural inclusions of the orthogonal summands where $R$ is realized and by $\pi_{k}: \mathbb{C}^{m} \rightarrow$ $\mathbb{C}^{a}$ the natural projections to them.

The operator $A_{k, l}: \mathbb{C}^{a} \rightarrow \mathbb{C}^{a}$, defined as $A_{k, l}=\pi_{l} \circ A \circ i_{k}$ transforms the irreducible representation $R$ in $\mathbb{C}^{a}$ to itself:

$$
A_{k, l} R=R A_{k, l}
$$

By Shur Lemma this symmetry operator $A_{k, l}$ is a scalar operator (of the multiplication by some complex number $\left.a_{k, l}\right)$.

The unitarity of operator $A$ implies that matrix $\left\{a_{k, l}\right\}$ is a unitary matrix. Any unitary matrix (of order $p$ ) defines a unitary operator,

$$
A=\sum_{k, l} i_{l} \circ A_{k, l} \circ \pi_{k}
$$

acting in $\mathbb{C}^{m}$ as a symmetry of the reducible representation $R^{\prime}$.

Therefore the dimension of the group $\{A\}$ of unitary symmetries of $R^{\prime}$ is equal to the dimension $p^{2}$ of the group of unitary matrices of order $p$, proving (the classical) Lemma 2 .

Both lemmas, together, provide the statement of Theorem 1: for $m_{k}=p_{k} a_{k}$ we find that the real dimension of the variety of unitary representations in $\mathbb{C}^{n}, n=\sum m_{k}$, which are unitary equivalent to $T$, is

$$
D=2 \sigma_{2}\left(m_{1}, \ldots, m_{s}\right)+\sum m_{k}^{2}-\sum p_{k}^{2}=n^{2}-\sum p_{k}^{2} .
$$

Corollary. The frequent representation in $\mathbb{C}^{n}$ has the minimal sum of squares of the multiplicities $f(p)=\sum p_{k}^{2}$ (among all integer points $p$ on the simplex $\sum a_{k} p_{k}=n$ ).

We have thus to study the integral points of this simplex, which is the closest integral point to the origin.

\section{Construction of the Frequent representation}

Neglecting the belonging of $p$ to the integral lattice, we find the real constrained minimum of $f$ from the collinearity of the gradient of $f$ to that of the constraint:

$$
\left(p_{1}, \ldots, p_{s}\right)=\lambda\left(a_{1}, \ldots, a_{s}\right)
$$

therefore the constrained minimum is attained at the point $p$ with coordinates

$$
p_{k}=\frac{a_{k} n}{\sum a_{k}^{2}}
$$


If this is an integral point (for instance, if the dimension $n$ is divisible by $\sum a_{k}^{2}$ ) then it defines the required frequent representation: $T_{k}$ should be repeated $p_{k}$ times.

For instance, the fundamental representation of a group in the space of functions on that group is frequent, since its dimension $n$ is equal to $\sum a_{k}^{2}$.

In the general case of fractional $p_{k}$, one should deform the rational point $p$ replacing it by an integral point $P$ belonging to the same hyperplane $(a, P)=n$.

First we replace $p_{k}$ by its integral part, $q_{k}=\left[p_{k}\right]: p_{k}=q_{k}+\left\{p_{k}\right\}, 0 \leq\left\{p_{k}\right\}<1$.

The point $q$ does not belong to the required hyperplane, namely we get the integral distortion

$$
n-\sum a_{k} q_{k}=\sum a_{k}\left\{p_{k}\right\}=a .
$$

One of the irreducible representations (say, $T_{1}$ ) is the trivial one-dimensional representation, $a_{1}=1$. To kill the distortion, add the integer $a$ to the multiplicity $q_{1}$ : the point $Q$ with coordinates

$$
Q_{k}=q_{k} \quad(k>1), \quad Q_{1}=q_{1}+a,
$$

lies on the required hyperplane, and is not far from the real minimum:

$$
\sum\left|Q_{k}-p_{k}\right| \leq s-1+\sum a_{k}
$$

Theorem 2. The frequencies $t_{k}$ of the irreducible unitary representations $T_{k}$ of dimensions $a_{k}$ (forming a frequent representation in $\mathbb{C}^{n}$ ) divided by $n$, converge to the coordinates of the point $p / n$ when $n$ grows to infinity.

These limits are proportional to the dimensions $a_{k}$,

$$
\lim _{n \rightarrow \infty} t_{k} / n=\frac{a_{k}}{\sum a_{k}^{2}}
$$

Proof. The distance between the real constrained minimum point $p$ and the integral point $Q$ is bounded by a constant independent of $n$. Namely by $K=s-1+\sum a_{k}$.

Therefore, $\lim (Q / n)=\lim (p / n)$ for $n \rightarrow \infty$.

The integral point $P$, defining the frequent representation, can't be farer from $p$ than $Q$. Indeed, by Pitagoras theorem (for the sum $f$ of the squares of the coordinates)

$$
f(P)=f(p)+|P-p|^{2} .
$$

Therefore $f(P)$ would be larger than $f(Q)$ if the point $P$ of the hyperplane $(P, a)=n$ would be farer than $Q$ from $p$.

Therefore $|P-p| \leq K$, proving theorem 2 .

\section{Additive semigroups of natural numbers}

The method described above provides also the frequent representation among the representations which are combinations of some chosen irreducible representations of dimensions 
$a_{k}(k=1, \ldots, s)$, whose multiplicities should be found (we shall prove that they are asymptotically proportional to the dimensions $a_{k}$, for representations with large dimension $n$ ).

The difficulty is the possible absence of 1 among the numbers $a_{k}$, making impossible the transition from $q$ to $Q$ in the above's proof.

To overcome this difficulty we shall use a theorem of Sylvester on the semigroups of natural numbers.

Theorem $3_{s}$. Suppose that the natural numbers $\left(a_{1}, \ldots, a_{s}\right)$ have no common divisor (greater than 1). Then every natural number $b$, greater than or equal to some integer $K(a)$, is representable in the form of the combination

$$
b=x_{1} a_{1}+\cdots+x_{s} a_{s}
$$

with integral non negative coefficients $x_{k}$.

Silvester, [5], has proved Theorem 32 with the optimal value $k=\left(a_{1}-1\right)\left(a_{2}-1\right)$.

Theorem $3_{s}$ follows immediately from this, at least with rough constant

$$
K=K_{s}=2^{s-1} \prod_{k=1}^{s} a_{k} .
$$

Interesting questions on the weak asymptotics of the optimal constant $K$ are discussed in [2], [6], but this Frobenius's problem on the optimal constant is still unsolved even for $s=3$.

Proof of Theorem $3_{s}$. Denote by $d$ the maximal common divisor of the numbers $\left(a_{1}, \ldots, a_{s-1}\right)$. According to Theorem $3_{s-1}$, every integer $c$, greater or equal to $K^{\prime}=K_{s-1}\left(a_{1} / d, \ldots, a_{s-1} / d\right)$, is representable in the form

$$
c=\sum_{k=1}^{s-1} x_{k}\left(a_{k} / d\right) .
$$

Consider the following $d$ integers: $N-r a_{s}, r=0,1, \ldots, d-1$. the integers $d$ and $a_{s}$ being relatively prime, exactly one of these $d$ integers is divisible by $d: N-r a_{s}=d c$.

If the integer $c$ is greater or equal to $K^{\prime}$, we get the required representation:

$$
N=c d+r a_{s}=\sum_{k=1}^{s-1} x_{k} a_{k}+r a_{s} .
$$

To get $c \geq K^{\prime}$ it suffices for $N$ to be not too small:

$$
N-r a_{s} \geq d K^{\prime}, \quad N \geq d K^{\prime}+(d-1) a_{s}
$$

Theorem $3_{s}$ follows now from the inequality

$$
K_{s} \geq d K^{\prime}+(d-1) a_{s},
$$

which is always fulfilled for $a_{1} \leq \ldots \leq a_{s}$. 
Indeed, this inequality follows from the evident facts

$$
K_{s}=2 a_{s} K^{\prime} \geq d K^{\prime}+(d-1) a_{s},
$$

implied by the inequalities

$$
a_{s} \geq a_{s-1} \geq d, \quad K^{\prime} \geq a_{s-1} \geq d-1 .
$$

Theorem $3_{s}$ is thus proved (with the quoted constant $K=K_{s}$ ).

Remark. The representation $b=x_{1} a_{1}+\ldots+x_{s} a_{s}$ of Theorem $3_{s}$ implies the inequalities

$$
x_{k} \leq b / a_{k}
$$

since the variables are nonnegative.

\section{Frequent combinations of irreducible representations}

Consider a unitary representation $T$ of finite group in space $\mathbb{C}^{n}$, which is the orthogonal sum of irreducible representations $T_{k}$ of dimension $a_{k}$ with multiplicities $p_{k}$,

$$
n=\sum p_{k} a_{k}
$$

Theorem 3. The most frequent representation of this class has the multiplicities $P_{k}$ of the irreducible components which are asymptotically proportional to the dimensions of these components:

$$
\lim _{n \rightarrow \infty} \frac{P_{k}}{n}=\frac{a_{k}}{\sum\left(a_{k}^{2}\right)} .
$$

Remark. If the dimensions $a_{k}$ have a common divisor $d$ then the dimension $n$ is supposed to be divisible by $d$ here.

Proof. Case $d>1$ is easily reducible to case of relatively prime dimensions $a_{s}(d=1)$, in which Theorem 3 is proved below.

It had been proved in $\S 1$ that the required integral point $P$ of the hyperplane $\sum P_{k} a_{k}=$ $n$ is the minimum point of the quadratic form $f=\sum P_{k}^{2}$ on the integral points of this hyperplane.

We start from the rational real constrained minimum point

$$
p_{k}=\frac{a_{k} n}{\sum a_{k}^{2}} \quad(k=1, \ldots, s)
$$

We approximate the point $p$ by the integral point $q$ with coordinates $q_{k}=\left[p_{k}\right]$, which does not belong to our hyperplane. The integral number

$$
\sum q_{k} a_{k}=n-\sum a_{k}\left\{p_{k}\right\}
$$

differs from $n$ by an integral number

$$
c=\sum a_{k}\left\{p_{k}\right\}<A, \quad A=\sum_{k=1}^{s} a_{k} .
$$


Chose the integral point $Z$ with coordinates $Z_{k}=q_{k}-K$. At this point the distance from our hyperplane is measured by the integral number

$$
\sum a_{k} Z_{k}=n-b, \quad b=c+K A
$$

The number $b$ is greater than or equal to $K$ (and is smaller than or equal to $(K+1) A$ ).

Choosing for $K$ the values $K_{s}$ of the proof of Theorem 3, we obtain from this theorem an integral vector $Y$ with nonnegative components $Y_{k}(k=1, \ldots, s)$, for which

$$
\sum a_{k} Y_{k}=b, \quad Y_{k} \leq b / a_{k} \leq(K+1) / A
$$

The integral point

$$
Q=Z+Y, \quad Q_{k}=q_{k}-K+Y_{k},
$$

belongs to the required hyperplane:

$$
\sum Q_{k} a_{k}=n
$$

The distance of this point to the real constrained minimum point is

$$
\begin{aligned}
R= & |Q-p| \leq|Q-Z|+|Z-q|+|q-p| \leq \\
& \leq s[(K+1) A+K+1]=s(K+1)(A+1) .
\end{aligned}
$$

Therefore, the value

$$
f(Q)=f(p)+|Q-p|^{2}=n+R^{2}
$$

is attained by the sum $f$ coordinates's squares of the integral point $Q$ in the hyperplane $(Q, a)=n$.

The existence of this integer point $Q$ implies inequality $|P-p| \leq R$, for the point $P$ of multiplicities of the frequent representation.

The independence of $R$ on $n$ implies the asymptotic equality

$$
\lim _{n \rightarrow \infty} \frac{P-p}{n}=0
$$

proving Theorem 3 .

\section{Examples: symmetric groups}

For the symmetric group $S(n)$ of permutations of $n$ elements the irreducible representations are classified by the Young diagrams, that is by the partitions

$$
n=y_{1}+y_{2}+\ldots+y_{m}, \quad y_{1} \geq y_{2} \geq \ldots \geq y_{m}
$$

into natural summands $0<y_{k}$. 
A Young table of a Young diagram is a monotone numbering of the $n=\sum y_{k}$ places in that Young diagram,

$$
1 \leq y_{i, j} \leq y_{i}
$$

by the $n$ numbers $(1, \ldots, n)$.

Here the monotonicity conditions are

$$
y_{i, j+1} \leq y_{i, j}, \quad y_{i+1, j} \leq y_{i, j} .
$$

The dimension $a[Y]$ of the representation associated to the partition $Y$ is equal to the number of all possible Young tables which can be associated to the corresponding Young diagram.

Example. For $n=4$, the 5 partitions of 4 are

$$
4, \quad 3+1, \quad 2+2, \quad 2+1+1 \quad \text { and } \quad 1+1+1+1 .
$$

The numberings of the lines $(i=1,2, \ldots)$ are:

$$
\begin{aligned}
4 & :(4>3>2>1) \quad ; \quad a[4]=1 ; \\
3+1 & :(4>3>2,1),(4>3>1,2),(4>2>1,3) \quad ; \quad a[3+1]=3 ; \\
2+2 & :(4>3,2>1),(4>2,3>1) ; \quad a[2+2]=2 ; \\
2+1+1:(4>3,2,1),(4>2,3,1),(4>1,3>2) \quad ; \quad a[2+1+1]=3 ; & \\
1+1+1+1 & :(4,3,2,1) \quad ; \quad a[1+1+1+1]=1 .
\end{aligned}
$$

Therefore, there are $s=5$ irreducible representations whose dimensions are $a_{k}=1,3,2,3,1$; the sum $\sum a_{k}^{2}=24$ being the number of elements of the group $G=S(4)$.

Our results mean in this case that there are asymptotically 3 times more copies of the 3 -dimensional representation, associated to the partition $[3+1]$, than of the 1-dimensional representations, associated to the partition [4] and to the partition $[1+1+1+1]$.

For the symmetric groups $S(5), S(6)$ and $S(7)$, the partitions and the dimensions $a_{k}$ of the representation are also given in the following table. The line $N[Y]$ represents the frequency of the Young diagram $Y$ measured by the number of the elements of the symmetric group $S(n)$, whose cycles have the lengths $y_{k}, k=1, \ldots, m$.

$$
\begin{aligned}
& |S(3)|=6, \quad s=3, \\
& 6=1^{2}+2^{2}+1^{2}=2+3+1 . \\
& |S(4)|=24, \quad s=5, \\
& 24=1^{2}+3^{2}+2^{2}+3^{2}+2^{2}+1^{2}=6+8+3+6+1 .
\end{aligned}
$$

\begin{tabular}{|c||c|c|c|}
\hline $\mathrm{Y}$ & {$[3]$} & {$[2+1]$} & {$\left[1^{3}\right]$} \\
\hline \hline $\mathrm{a}[\mathrm{Y}]$ & 1 & 2 & 1 \\
\hline $\mathrm{N}[\mathrm{Y}]$ & 2 & 3 & 1 \\
\hline
\end{tabular}




\begin{tabular}{|c||c|c|c|c|c|}
\hline $\mathrm{Y}$ & {$[4]$} & {$[3+1]$} & {$\left[2^{2}\right]$} & {$\left[2+1^{2}\right]$} & {$\left[1^{4}\right]$} \\
\hline \hline $\mathrm{a}[\mathrm{Y}]$ & 1 & 3 & 2 & 3 & 1 \\
\hline $\mathrm{N}[\mathrm{Y}]$ & 6 & 8 & 3 & 6 & 1 \\
\hline
\end{tabular}

$$
\begin{gathered}
|S(5)|=120, \quad s=7 \\
120=1^{2}+4^{2}+5^{2}+6^{2}+5^{2}+4^{2}+1^{2}=24+30+20+20+15+10+1 .
\end{gathered}
$$

\begin{tabular}{|c||c|c|c|c|c|c|c|}
\hline $\mathrm{Y}$ & {$[5]$} & {$[4+1]$} & {$[3+2]$} & {$\left[3+1^{2}\right]$} & {$\left[2^{2}+1\right]$} & {$\left[2+1^{3}\right]$} & {$\left[1^{5}\right]$} \\
\hline \hline $\mathrm{a}[\mathrm{Y}]$ & 1 & 4 & 5 & 6 & 5 & 4 & 1 \\
\hline $\mathrm{N}[\mathrm{Y}]$ & 24 & 30 & 20 & 20 & 15 & 10 & 1 \\
\hline
\end{tabular}

$$
|S(6)|=720, \quad s=11
$$$$
720=2 \cdot 1^{2}+4 \cdot 5^{2}+2 \cdot 9^{2}+2 \cdot 10^{2}+16^{2}
$$$$
=120+144+90+90+40+120+40+15+45+15+1 \text {. }
$$

\begin{tabular}{|c||c|c|c|c|c|c|c|}
\hline $\mathrm{Y}$ & {$[6]$} & {$[5+1]$} & {$[4+2]$} & {$\left[4+1^{2}\right]$} & {$\left[3^{2}\right]$} & {$[3+2+1]$} & {$\left[3+1^{3}\right]$} \\
\hline \hline $\mathrm{a}[\mathrm{Y}]$ & 1 & 5 & 9 & 10 & 5 & 16 & 10 \\
\hline $\mathrm{N}[\mathrm{Y}]$ & 120 & 144 & 90 & 90 & 40 & 120 & 40 \\
\hline
\end{tabular}

\begin{tabular}{|c||c|c|c|c|}
\hline $\mathrm{Y}$ & {$\left[2^{3}\right]$} & {$\left[2^{2}+1^{2}\right]$} & {$\left[2+1^{4}\right]$} & {$\left[1^{6}\right]$} \\
\hline \hline $\mathrm{a}[\mathrm{Y}]$ & 5 & 9 & 5 & 1 \\
\hline $\mathrm{N}[\mathrm{Y}]$ & 15 & 45 & 15 & 1 \\
\hline
\end{tabular}

$$
|S(7)|=5040, \quad s=15,
$$

$$
\begin{aligned}
5040 & =2\left(1^{2}+6^{2}+2 \cdot 14^{2}+15^{2}+35^{2}+21^{2}\right)+20^{2} \\
& =720+840+2 \cdot 504+420+630+280+2 \cdot 210+420+105+70+105+21+1 .
\end{aligned}
$$

\begin{tabular}{|c||c|c|c|c|c|c|c|c|}
\hline $\mathrm{Y}$ & {$[7]$} & {$[6+1]$} & {$[5+2]$} & {$\left[5+1^{2}\right]$} & {$[4+3]$} & {$[4+2+1]$} & {$\left[3^{2}+1\right]$} & {$\left[4+1^{3}\right]$} \\
\hline \hline $\mathrm{a}[\mathrm{Y}]$ & 1 & 6 & 14 & 15 & 14 & 35 & 21 & 20 \\
\hline $\mathrm{N}[\mathrm{Y}]$ & 720 & 840 & 504 & 504 & 420 & 630 & 280 & 210 \\
\hline
\end{tabular}

\begin{tabular}{|c||c|c|c|c|c|c|c|}
\hline $\mathrm{Y}$ & {$\left[1^{7}\right]$} & {$\left[2+1^{5}\right]$} & {$\left[2^{2}+1^{3}\right]$} & {$\left[3+1^{4}\right]$} & {$\left[2^{3}+1\right]$} & {$\left[3+2+1^{2}\right]$} & {$\left[3+2^{2}\right]$} \\
\hline \hline $\mathrm{a}[\mathrm{Y}]$ & 1 & 6 & 14 & 15 & 14 & 35 & 21 \\
\hline $\mathrm{N}[\mathrm{Y}]$ & 1 & 21 & 105 & 70 & 105 & 420 & 210 \\
\hline
\end{tabular}

Here $1^{3}$ means $1+1+1$ and similarly for $1^{i}$.

It seems that the shape of the Young diagram $Y$ of the representation of largest dimension is not far from the Vershik's asymptotics for the lengths $n_{i}$ of the cycles of most permutations of a large set $(n \rightarrow \infty)$ (which provides for $n_{i}$ the equation $h\left(n_{i}\right)+h(i)=$ const, with some function $h$ calculated explicitly).

But I had not verified this conjecture and the resulting asymptotic behavior of the maximal $a[Y]$ and $N[Y]$ for $n \rightarrow \infty$. 


\section{$7 \quad$ Formula for the dimension of representations of $S(n)$}

To write the general formula for the dimension of the representation of the symmetric group $S(n)$, which is associated to the partition

$$
n=m_{1}+m_{2}+\ldots+m_{s}, \quad m_{1} \geq m_{2} \geq \ldots \geq m_{s}
$$

instead of the lengths, $m_{k}$, of the lines, we shall use the lengths of the corresponding hooks defined as $h_{k}=m_{k}+(s-k)$ :

$$
h_{1}=m_{1}+s-1, h_{2}=m_{2}+s-2, \ldots, h_{s}=m_{s}, h_{1}>h_{2}>\ldots>h_{s} .
$$

Theorem 4. The dimension of the irreducible representation associated to the given partition is equal to

$$
a\left(m_{1}, \ldots, m_{s}\right)=\frac{\left(m_{1}+m_{2}+\ldots+m_{s}\right) !}{F\left(h_{1}, h_{2}, \ldots, h_{s}\right)},
$$

the denominator being

$$
F(h)=\frac{h_{1} ! h_{2} ! \ldots h_{s} !}{\prod\left(h_{i}-h_{j}\right)}
$$

(the product has $s(s-1) / 2$ terms: $s \geq i . j \geq 1$ ).

These numbers $a\left(m_{1}, \ldots, m_{s}\right)$ are strange extensions of the Catalan numbers $1,2,5,14$, $42, \ldots$ (the $n$-th Catalan number counts all possible triangulations of a convex $(n+2)$-gon by its diagonals).

Examples: $a(m)=a(m, 1)=m, a(m, 2)=(m+2)(m-1) / 2$,

$$
a(m, n)=(m+2) \ldots(m+n)(m-(n-1)) /(n !)
$$

(Catalan numbers being $a(m, m)=a(m, m-1)$ ).

For Young diagrams with 3 lines we get the following extended Catalan numbers:

$$
\begin{aligned}
& a(m, n, 1)=(m+3) \ldots(m+n+1)(m+1)(m-(n-1)) /((n+1) ! / n), \\
& a(m, n, 2)=(m+3) \ldots(m+n+2) m(m-(n-1)) /(2(n+1) ! /(n-1)), \\
& a(m, n, k)=(m+3) \ldots(m+n+k)(m+2-k)(m-(n-1)) / F_{k}(n),
\end{aligned}
$$

where $F_{k}(n)=k !(n+1) ! /(n+1-k)$.

For $s>3$ lines Theorem 4 is still applicable, providing, for instance,

$$
a(m, n, 1,1)=(m+4) \ldots(m+n+2)(m+2)(m+1)(m+1-n) / G(n),
$$

where $G(n)=2(n+2)(n-1) !$.

Theorem 4 contains a lot of formulae, like

$$
a(m, 3,2,1)=(m+6)(m+5)(m+4) m(m-2) / 45 .
$$


The proofs of all these formulae start from the classification of the Young tables, according to the place occupied in the Young diagram by the smallest element, 1. It might take $s$ positions for a diagram of $s$ lines (at the end of any line). The resulting identity for the numbers of Young tables is the recurrent relation

$$
a\left(m_{1}, m_{2}, \ldots\right)-a\left(m_{1}-1, m_{2}, \ldots\right)=\sum_{k=2}^{s} a\left(m_{1}, m_{2}, \ldots, m_{k-1}, m_{k}-1, m_{k+1}, \ldots, m_{s}\right) .
$$

In this formula the term

$$
a_{k}=a\left(m_{1}, m_{2}, \ldots, m_{k-1}, m_{k}-1, m_{k+1}, \ldots, m_{s}\right)
$$

is considered as being zero, if the arguments are not verifying the monotonicity condition (that is, in the case where $m_{k}-1<m_{k+1}$, which happens if $m_{k}=m_{k+1}$ ).

The above recurrent relation shows that $a\left(m_{1}, m_{2}, \ldots, m_{s}\right)$ is a polynomial of degree $N+1$ in $m_{1}$, provided that the summands $a_{k}$ have degrees at most $N$ in $m_{1}$.

Since for the diagram with only one line $a\left(m_{1}\right)=1$ is a polynomial of degree 0 , we find all the degrees (in $\left.m_{1}\right): \operatorname{deg} a\left(m_{1}, m_{2}, \ldots, m_{s}\right)=m_{2}+m_{3}+\ldots+m_{s}$. Moreover, the recurrent relation provides for the required polynomial $a\left(m_{1}, \ldots\right)$ an explicit equation from the explicit equations of the $a_{k}$ polynomials of smaller degree and from the boundary condition $a\left(m_{2}-1, m_{2}, m_{3}, \ldots, m_{s}\right)=0$ at $m_{1}=m_{2}-1$.

To prove the formulae of Theorem 4, it suffices therefore to check that these formulae satisfy the recurrent equation. This identity is not too difficult to prove directly, but I shall show the simpler arguments, useful to calculate the denominators $F$ in Theorem 4 and, $F_{k}$ and $G$ in the examples. The checking of a ready formula is much easier than the guessing of its form, which is rather provided by the examples above.

Namely, suppose that the leading term of a polynomial $a$ in $m_{1}$ is

$$
a\left(m_{1}, \ldots\right)=m_{1}^{p} / f\left(m_{2}, \ldots, m_{s}\right),
$$

the degree being $p=m_{2}+\ldots+m_{s}$.

The leading terms of the recurrent relation at the left hand side and at the right hand side are then

$$
p m_{1}^{p-1} / f\left(m_{2}, \ldots, m_{s}\right)
$$

and

$$
m_{1}^{p-1} \sum_{k=2}^{s}\left(1 / f_{k}\left(m_{2}, \ldots, m_{s}\right)\right),
$$

where $f_{k}\left(m_{1}, \ldots, m_{s}\right)=f\left(m_{2}, \ldots, m_{k-1}, m_{k}-1, m_{k+1}, \ldots, m_{s}\right)$.

We get therefore the recurrent relation for the denominator $f\left(m_{2}, \ldots, m_{s}\right)$ :

$$
\frac{p}{f}=\sum_{k>1} \frac{1}{f_{k}}
$$


defining $f(m)$, provided that it is known for the smaller arguments involved in the values of $f_{k}$.

This relation leads to the values of the denominators in the examples following Theorem 4 and then recurrently to the expression of the denominator $F$ in Theorem 4.

The elementary identity, proving that formulae of Theorem 4 verify the above recurrent relation, is reducible to some peculiar combinatorial facts (being a version of Euler-Jacobi identity).

Lemma 3. The following identity holds

$$
\sum_{k=1}^{s}\left(h_{k} \prod_{i \neq k}\left(1+\frac{1}{h_{i}-h_{k}}\right)\right)=\sum_{k=1}^{s} m_{k}
$$

(the last sum being equal to $\left.\left(\sum_{k=1}^{s} h_{k}\right)-s(s-1) / 2\right)$.

Each product here contains $s-1$ multipliers.

Example. For $s=2$ Lemma 3 is evident:

$$
a\left(1+\frac{1}{b-a}\right)+b\left(1+\frac{1}{a-b}\right)=a+b+\frac{a-b}{b-a}=a+b-1
$$

To prove Lemma 3, represent the left hand side sum as the sum of its homogeneous parts, $R_{0}+R_{1}+\cdots$, where $R_{0}=\sum h_{k}$, while for $r>0$

$$
R_{r}\left(h_{1}, \ldots, h_{s}\right)=\sum\left(\frac{h_{k}}{\prod_{p=1}^{r}\left(h_{i_{p}}-h_{k}\right)}\right) .
$$

Tanking into account the ranges of $k$ and of $i$, this sum contains $s C_{s-1}^{r}$ terms, since $1 \leq k \leq s$ and the sets $\left\{i_{p}\right\}$ are all the subsets of $r$ elements of the set (of $s-1$ elements) $\{1, \ldots, s\} \backslash\{k\}$. The calculation of the sum $R_{r}$ is also a version of Euler-Jacobi identity. Namely we get

Lemma 4. The first order contribution is equal to a constant, $R_{1}=-s(s-1) / 2$, while all higher order contributions vanish: $R_{r}=0$ for $r>1$.

Proof. The $r+1$ elements $\left(k, i_{1}, \ldots, i_{r}\right)$ provide $r+1$ terms in the expression of $R_{r}$, according to the choice of the element playing the rôle of $k$.

These $r+1$ terms provide all together the contribution $R_{r}\left(h_{k}, h_{i_{1}}, \ldots, h_{i_{r}}\right)$. Therefore, we proved the representation of $R_{r}$ in terms of a function of only $r+1$ variables,

$$
R_{r}(h)=\sum R_{r}\left(h_{k}, h_{i_{1}}, \ldots, h_{i_{r}}\right)
$$

There are $C_{s}^{r+1}$ terms in this sum. For $r=1, s=2$ we get an evident relation

$$
R_{1}(a, b)=\frac{a}{b-a}+\frac{b}{a-b}=-1
$$


therefore $R_{1}(h)=-C_{s}^{2}=-s(s-1) / 2$ for any $s$.

To prove Lemma 4, it remains to prove the vanishing of the function of $r+1$ variables

$$
R_{r}\left(h_{1}, \ldots, h_{r+1}\right), \quad r>1, \quad(\text { corresponding to } s=r+1) \text {. }
$$

This function is, by its definition,

$$
R_{r}=\sum_{k=1}^{s} \frac{h_{k}}{\prod_{i \neq k}\left(h_{i}-h_{k}\right)} .
$$

Denote by $f$ the following polynomial of degree $s$

$$
f(h)=\prod_{j=1}^{s}\left(h_{j}-h\right) .
$$

In terms of this polynomial one represents $R_{r}$ in the form of the sum of residues

$$
R_{r}=(-1)^{s-1} \sum_{j=1}^{s} \frac{h_{j}}{f^{\prime}\left(h_{j}\right)}
$$

This sum of residues vanishes for $s>1$ (the form $d h / f(h)$ being in this case regular at infinity).

This ends the prof of Lemma 4, hence of Lemma 3, and leads to Theorem 4, by a chain of recurrences, as it is explained above.

Formulae of Theorem 4 define integer polynomials $A$ of degree $p=m_{2}+\cdots+m_{s}$ in variable $m_{1}$ and also define integer denominators $G$, providing the the dimensions of the representations in the form of the values of the rational function,

$$
a\left(m_{1}, \ldots, m_{s}\right)=\frac{A}{G} .
$$

The polynomials $A$ are defined by the formulae of Theorem 4 everywhere, including the negative values of the arguments. For $m_{1} \geq m_{2} \geq \ldots \geq m_{s} \geq 0$, those polynomials have clear combinatorial meaning counting the Young tables (in the Young diagram, defined by the partition $\left.n=m_{1}+m_{2}+\cdots+m_{s}\right)$.

The combinatorial meaning of the values at the other points would be interesting, specially due to the peculiar distributions of the roots of the polynomials $A$. The fact, that these $p$ roots are always integers, have no a priory proof, being just a strange and unexpected result of long explicit calculations, which might be avoided if these roots were predicted using the combinatorial arguments of the Young diagrams of negative length.

\section{References}

[1] Arnold, V. I., Modes and quasimodes. Functional Analysis and its Applications, 1972, vol. 6 N 2, pp 12-20. 
[2] Arnold, V. I., Weak asymptotics of the numbers of the solutions of Diophantine problems. Functional Analysis and its Applications, 1999, vol. 33 N 3, pp. 65-66.

[3] Arnold, V. I., On evolution of magnetic field under the action of the transport and of diffusion, in "Some problems of modern calculus" To the memory of V.M. Alexeev, V.M. Tikhomirov Editor, Moscow, MGU, 1984, pp. 8-21.

[4] Arnold, V. I., Remarks on perturbation problems of Mathieu type. Russian Mathematical Surveys, 1983, vol. 38 N 4, pp. 189-203.

[5] Sylvester, J.J., Mathematical questions with their solutions. Educational Times, 1884, vol. 41, pp. 21.

[6] Arnold, V. I., Problems. Phasis, 2000, Problem 1999-8, pp. 141-142 and 447-449. 